\title{
Ubiquitous modelling: In honour of Tony Starfield's 70th anniversary
}

AUTHORS:

David P. Mason ${ }^{1}$

Michael Sears ${ }^{2}$

Anthony M. Starfield ${ }^{3}$

\section{AFFILIATIONS:}

${ }^{1}$ Professor Emeritus, School of Computational and Applied Mathematics, University of the Witwatersrand, Johannesburg, South Africa

${ }^{2}$ Visiting Professor, School of Computer Science, University of the Witwatersrand, Johannesburg, South Africa

${ }^{3}$ Professor Emeritus, University of Minnesota, St Paul,

Minnesota, USA

\section{CORRESPONDENCE TO:}

Michael Sears

EMAIL:

michael.sears@wits.ac.za

\section{POSTAL ADDRESS:}

School of Computer Science, University of the Witwatersrand, Private Bag 3, Wits 2050, South Africa

\section{KEYWORDS:}

ecological modelling; marine modelling; rhino conservation; mathematical modelling; quantitative reasoning

\section{HOW TO CITE:}

Mason DP, Sears M, Starfield AM. Ubiquitous modelling: In honour of Tony Starfield's 70th anniversary. S Afr J Sci. 2014;110(5/6), Art. \# a0067, 5 pages. http://dx.doi. org/10.1590/sajs.2014/a0067

(C) 2014. The Authors. Published under a Creative Commons Attribution Licence.

\begin{abstract}
A 1-day symposium on Computational and Mathematical Modelling was held on 21 April 2012 in the School of Computational and Applied Mathematics at the University of the Witwatersrand in honour of Tony Starfield on the occasion of his 70th anniversary. Starfield was Professor of Applied Mathematics at the University of the Witwatersrand from 1969 to 1979 . He spent his career in teaching and doing research in computational and mathematical modelling. The unifying theme of the symposium was computational and mathematical modelling in the broadest sense. Starfield's 60th anniversary was celebrated by a symposium for which the presentations were made by his colleagues of long standing. The proceedings of that symposium were published in the South African Journal of Science as the Starfield Festschrift. 1 The symposium on Starfield's 70th anniversary looked to the future of mathematical and computational modelling in the 21 st century, with an emphasis on the contributions of a new generation of modellers.
\end{abstract}

Starfield's keynote paper - 'Ubiquitous modelling' - opened the symposium. He reviewed the development of modelling over the past 50 years; the ideas expressed in his paper were well illustrated by the symposium itself.

\section{Ubiquitous modelling}

Seventy years ago one might have defined modelling as the use of mathematics to analyse problems; in other words, applied mathematics. The portfolio of modelling problems would have been dominated by theoretical physics and mechanics. Mathematical techniques determined both the problems selected and the way in which they were posed; there was no point in attempting to model a problem that was mathematically 'intractable'. Advances in techniques (Fourier series, Laplace transforms) opened up new modelling opportunities, but almost all of them within the same neighbourhood of mechanics and theoretical physics.

Modelling problems in what we would now call Operations Research became important during the Second World War and drove the need for more computational power. The development of computers, in turn, revolutionised modelling. At first this revolution was seen as an extension of traditional modelling: numerical techniques and computers could be used to tackle problems that had previously been mathematically intractable. A numerical solution was, however, viewed as an inferior analysis and some journals were slow to publish computational results. I recall J.C. Jaeger, one of the great applied mathematicians of the 20th century, describing the finite element method at a conference in the early 1970 s as 'an expensive way to get inaccurate results to irrelevant problems'. Within a few years, finite elements were an indispensable tool in engineering analysis.

The Department of Applied Mathematics at the University of the Witwatersrand was in the forefront of the modelling revolution during the 1970 s, beginning with the development of numerical solutions to tackle problems in the traditional portfolio, but recognising very quickly that the real revolution was in exploiting computational power to expand the modelling portfolio into unexplored disciplines. Modelling seminars were started to explore collaborative opportunities in medicine, biology, ecology, wildlife management, building science, socio-economics, and traffic engineering - in other words, ubiquitous modelling!

Computers created the opportunity to explore new disciplines. New disciplines brought new and unusual modelling problems. New problems plus computer power engendered new modelling approaches. New approaches opened up the way for further new problems. The co-evolution of computers, modelling techniques and ubiquitous modelling has been rapid and exciting. Spatial modelling, neural networks, agent-based modelling, visualisation - these are just some of the techniques that a modeller can access using software that is getting easier and easier to use.

But has the philosophy of modelling kept pace with this rapid expansion? If modelling was once constrained by mathematical tractability, that constraint also provided a paradigm for modelling methodology. A modeller knew that it was usually impossible to solve the 'real' problem. The secret was to find a similar problem that was tractable, and then to interpret the results in the light of differences between the real problem and the model. Everybody understood the difference between the 'real world' and the 'model world' and this provided a framework for both appreciating and criticising a modelling paper or report. Moreover, because the number of mathematical techniques was limited, modellers could easily comprehend what other modellers had done. There was a recognised discipline to modelling. Life in the frontier towns of ubiquitous computational modelling is exciting and full of opportunities, but it has produced both good and bad models and the discipline has been slow to follow the pioneers.

What is the discipline of ubiquitous modelling as opposed to mathematical modelling? Jaeger's comment on finite elements was really a call for modelling discipline: when it is possible to model almost anything in a number of different ways, one has to ask whether time and money have been well spent, whether the modelling results fit the problem being addressed, and whether the problem has been carefully framed. The minimal set of questions to be asked about any modelling exercise is:

- Is the 'real world' objective of the exercise clear and well-defined?

- Has the model world been carefully and parsimoniously designed and have all assumptions been noted?

- Is the solution method appropriate, understandable and reproducible?

- Have the results been interpreted back to the real world? 
- And, last but certainly not least, how robust are the conclusions? Has a sensitivity analysis explored uncertainties in the data and has an assumption analysis considered the effects of the key assumptions?

The word 'discipline' has been used above in the sense of a modelling methodology, but what about a modelling discipline in the sense of an academic subject? Starfield and Salter ${ }^{2}$ argue that the methodology is the academic discipline; the above list of questions provides the core of what needs to be taught in any attempt to introduce students to the subject of modelling. Their paper was written in the context of a concerted effort at Oberlin College to introduce modelling across the undergraduate curriculum - ubiquitous modelling - and draws on their experiences in teaching introductory modelling classes to students with backgrounds ranging from literature to neuroscience.

That leads to three important questions:

1. Why should modelling be taught ubiquitously?

2. Can it be taught to students with weak mathematical and numerical skills?

3. How should it be taught?

To provide short answers in reverse order:

Starfield and Salter expand in their 2010 paper $^{2}$ on ways to teach the core methodology of modelling. That these can be taught is demonstrated by the success of their classes and is caught by the following (paraphrased) comment of an English major:

\begin{abstract}
My friends asked me, over coffee, what classes I was taking. I mentioned Modelling and they burst out laughing and started imagining me showing off designer clothes. It struck me that mannequins who model clothes really are models in the sense that they are designed parsimoniously to achieve a clear objective - to show the clothes to best effect.
\end{abstract}

But why should modelling be taught ubiquitously? Firstly, the questions at the core of modelling methodology are in fact at the core of critical thinking in general and experimental science in particular. Moreover, modelling is easy to teach in a way that instantaneously illustrates the power of this methodology. Secondly, we all live in a world that cries out for systematic thinking. One cannot make thoughtful decisions without understanding the interactions and trade-offs between multiple factors. At the very least, to be a responsible citizen, one has to have the ability to ask critical questions about modelling results.

\section{Symposium overview}

The application areas addressed by the presentations were wide ranging, involving marine models, land-based ecological models, models in astronomy and astrophysics and models from South African industry. In addition, a panel discussion was held as part of the symposium on rhino conservation in southern Africa as an example of a problem that requires systematic thinking.

Modelling approaches included spatial models, simulation models, and agent-based models as well as mathematical models. The breadth of the papers, both in terms of application area and in terms of methodology, illustrated the idea of ubiquitous modelling in a remarkable way. We present the summary by subject area as that was the order in which the papers were presented. Table 1 summarises the spread of the papers in terms of methodology and approach. (Some of the presentations appear more than once in the table because they covered a variety of models or used multiple approaches.)

\section{Marine process models}

The first session was concerned with marine process modelling. Starfield has collaborated for many years with the Marine Research (Ma-Re) Institute at the University of Cape Town.

\section{Modelling plankton production}

John Field (former Director of the Ma-Re Institute) presented a paper entitled 'Modelling plankton production by combining sub-surface shipboard measurements with those of the ocean surface from satellites' in which he described the process of developing models that take into account large data sets of daily ocean surface estimates of wind, temperature and chlorophyll from satellites and combining these with sparse ship-based depth profiles of temperature and chlorophyll., 3.4 The models are based on current understanding of the main physical, chemical and biological processes involved in the variability of phytoplankton growth. The temperature and chlorophyll profiles were clustered into a manageable number of optical profiles and dynamical neural networks were then used to predict which profile will occur at a particular place and season, given a few days of surface estimates of wind, temperature and chlorophyll. These profiles are used to give monthly estimates of plankton production over wide areas of the coastal and open ocean.

\section{Starfield ripples in marine social-ecological}

\section{systems research}

Astrid Jarre (SA Research Chair in Marine Ecology \& Fisheries, Ma-Re Institute) spoke on behalf of her research team. Their focus is on how to cope with global change in marine social-ecological systems. She described how Starfield had stimulated modelling research in this area, with reference to at least seven projects that were influenced by his modelling philosophy and, in some cases, his modelling methodology for involving both academics and non-academics in conservation management decisions. Examples are: a (system) model of penguin population dynamics in relation to various pressures; a frame-based model looking at different states of the Benguela ecosystem ${ }^{5,6}$; expert systems for ecosystem-based fisheries management; and modelling at the interface between social sciences and fisheries ecology. As an example of ubiquitous modelling, Jarre described how the parsimony and elegance of Starfield's modelling approach had even won the respect of an anthropologist in the group who used Starfield's rapid prototyping philosophy in his MSocSci thesis research.

\section{Modelling in deep sea sonar}

Michael Mitchley (Computer Science, University of the Witwatersrand) examined the use of high resolution beam forming methods for sonar bathymetry, including techniques for full-rank covariance matrix estimation and signal enumeration. The modelling of both passive and active sonar was discussed and current challenges were highlighted.

\section{Land-based ecological models}

The second session was concerned with ecological modelling of animals on land.

\section{Stability and resilience in seasonal oscillators}

Norman Owen-Smith (former Leader of the Centre for African Ecology, School of Animal, Plant and Environmental Sciences, University of the Witwatersrand) presented a paper entitled 'Stability and resilience in seasonal oscillators: Towards mechanistic population models'. OwenSmith began by observing that models of population dynamics have become fossilised in phenomenological forms thathave dubious predictive value, for example, logistic and allied density-dependent equations, Lotka-Volterra models of coupled consumer-resource interactions and Leslie and other matrix models for structured populations. All are unsatisfactory when the reality of global environmental change raises the need for more reliable models to guide difficult decisions for biodiversity conservation. ${ }^{7} \mathrm{He}$ explored how these alternative model forms might be reconciled and adjusted to represent more faithfully the mechanisms generating changes in population abundance. Populations can be viewed as dissipating systems capturing material and energising resources to maintain their organisation, despite environmental perturbations. The problem for herbivores is that the resource supply varies over the annual 
Table 1: Classification of presentations at the workshop

\begin{tabular}{|c|c|c|c|}
\hline Approach & Model type & Application area & Presenter \\
\hline Agent based & Economic model & Fisheries system dynamics & A Jarre \\
\hline Expert system & Computer model & Marine ecosystem dynamics & A Jarre \\
\hline Frame based & Computer model & Marine ecosystem dynamics & A Jarre \\
\hline Network model & Representative model & Robotics & D Fanucchi \\
\hline Neural network & Representative model & Population dynamics & J Field \\
\hline Simulation & Computer model & Population dynamics & A Jarre \\
\hline Simulation & Computer model & Ecosystem & D Richardson \\
\hline Simulation & Computer model & Chemical engineering & A Hutchinson \\
\hline Simulation & Physical model & Mining engineering & R Kgatle \\
\hline Simulation & Physical/computer model & Sonar & M Mitchley \\
\hline Statistical correlation & Physical model & Astrophysics & A Tailor \\
\hline Statistical extrapolation & Representative model & Population dynamics & J Field \\
\hline Theoretical & Computational model & Population dynamics & N Owen-Smith \\
\hline Theoretical & Mathematical model & Population dynamics & J Shaw; all \\
\hline Theoretical & Physical model & Astrophysics & R Herbst \\
\hline Theoretical & Physical model & Mining engineering & G Fareo \\
\hline Theoretical & Economic model & Population dynamics & J Shaw; all \\
\hline
\end{tabular}

cycle because plant growth is seasonally phased. How then do herbivore populations persist despite seasonal as well as annual variation in the resource supply? This question requires judicially investing the surplus gains of the good times to promote persistence through the lean times. Owen-Smith suggested how this intrinsically disequilibrial system might be simulated by adopting a metaphysiological approach employing a currency of biomass rather than numerical population density. Thereby insights are generated into strategies of resource allocation and adaptive selection among functionally distinct resource types at different stages of the seasonal cycle. Owen-Smith then threw out the challenge: how might such a model system be generalised mathematically and treated analytically?

\section{Modelling the dynamics of complex systems in a highly variable environment}

David Richardson (Mathematics and Applied Mathematics, University of Cape Town) began by describing semi-arid rangelands as hierarchical systems with many levels of organisation, ranging from tissues of plants and animals to populations at the ecosystem level. This ecosystem is driven by rainfall which varies widely both between and within years. Rangeland vegetation also varies in both botanical and chemical composition and the spatial distribution of edible material on a plant varies between species. Consequently, mechanisms controlling diet selection and intake differ between veld types. Furthermore, the relative importance of producing meat, milk and fibre varies with the objectives of the pastoralists and with the ecosystem. Variables at one level influence processes at both higher and lower levels. Processes in the different levels operate on widely different timescales and long-term changes depend on the effects of short-term processes. A major difficulty frequently encountered in modelling rangelands is the lack of empirical information between animal productivity, vegetation and rainfall. ${ }^{8}$

Richardson's approach was to simulate the different levels of the system separately and use model output of one level as input for other levels. Model output showed how rainfall variability within years can influence the long-term behaviour of the system and also explained observed unexpected results such as accepted 'good management' leading to a greater probability of rangeland degradation.

\section{How can modelling help save the rhino?}

Jo Shaw (Large Mammal Trade, Traffic East/Southern Africa) gave this presentation, which served as a good introduction to the panel discussion and group meetings on rhino. Since 2008, the number of rhinos poached in South Africa has risen dramatically year on year with a total of 448 animals killed during 2011. Rhino horn has been used for thousands of years as a fever-reducing agent as part of traditional Chinese medicine practices. However, research by TRAFFIC, the wildlife trade monitoring network, indicates that more recently there has been increased demand from Vietnam, where rhino horn has a high status cachet. There are 
approximately 19000 white rhinos and 1900 black rhinos in South Africa and rhino numbers continue to increase, with births exceeding total mortalities. However, if rhino deaths from poaching continue to rise at the current rate, some scientists estimate that rhino populations will begin to decline by 2015 . It is thought that $25 \%$ of rhinos in South Africa are privately owned, but accurate data on live rhino numbers and rhino horn stockpiles can be very difficult to obtain. This presentation provided an overview of current understanding of the rhino poaching crisis and raised research questions that could be investigated using computer modelling exercises to inform rhino conservation actions and political decision-making.

\section{Rhino conservation}

Trade in rhino horn is prohibited by CITES (Convention on International Trade in Endangered Species). However, illegal trading takes place and the price of rhino horn is believed to be about USD65 000 to USD70 000 per kilogram. A rhino horn weighs about $4-5 \mathrm{~kg}$. The horn can be removed from the rhino down to about $20 \mathrm{~mm}$ above the base of the horn without harming the rhino. The horn grows back at a rate of about $40 \mathrm{~mm}$ per year.

A panel consisting of Tony Starfield, Norman Owen-Smith, Jo Shaw and David Cumming, formulated three broad modelling questions for small groups of participants to consider:

1. Can a sustainable legal trade capture the market?

2. How can stockpiled rhino horn be used strategically to supply the market?

3. How can a production model for rhino horn, including an economic component, be constructed?

The participants split up into three groups, with each group tackling one question. The groups were not asked to formulate the models mathematically at that stage.

Rhino conservation is essentially a problem in environmental economics. This short exercise framed several plausible modelling projects within the context of environmental economics, which led to the consideration of this problem at the Tenth Mathematics in Industry Study Group which was held at the University of the Witwatersrand from 14 to 18 January $2013 .{ }^{9}$

\section{Models in astronomy and astrophysics}

This session contained two presentations on models in astronomy and astrophysics by master's students at the University of the Witwatersrand.

\section{Gravitational torques of nearby barred spiral galaxies}

Asha Tailor's research was aimed at using quantitative methods in classifying barred galaxies. Maximum relative gravitational torques were derived and compared for a sample of 40 nearby bright barred disk galaxies. Torques were compared between galaxy pairs and excellent agreement was found between the $3.6 \mu \mathrm{m}$ and $4.5 \mu \mathrm{m}$ morphology. The sample used incorporated a wide range of inclination and bar strength values. The tight coupling of $3.6 \mu \mathrm{m}$ and $4.5 \mu \mathrm{m}$ morphology provides an excellent opportunity to classify intermediate redshift galaxies out to $z=0.25$. This has important implications for bar-fraction estimates and galaxy accretion and evolution models.

\section{Stellar models}

Rhameez Herbst investigated the effect of mass on the radiation flux from a relativistically rotating neutron star. A pulsar was modelled by simulating a relativistically rotating magnetic dipole embedded within a neutron star. The resulting equations retain the mass of the neutron star thereby introducing effects of general relativity on the radiation from the dipole. Exact solutions to the modelling equation were presented as well as plots of energy spectra at different rotational velocities and inclination angles. The results demonstrated that the high speed enhancement of the radiation is always more than compensated for by the frame dragging effect for relativistic neutron star masses leading to a net reduction of radiation from the star. ${ }^{10}$

\section{Models from South African industry}

The remaining presentations were based on problems from South African industry and were given by postgraduate students from the School of Computational and Applied Mathematics at the University of the Witwatersrand. The problems had been submitted to one of the Mathematics in Industry Study Group meetings held annually in South Africa since 2004.

\section{Extraction of sugar from shredded cane in a diffuser}

The extraction of sugar from shredded cane takes place in a diffuser. ${ }^{11}$ A diffuser generally has 12 to 14 components. Ashleigh Hutchinson presented a model in which the sugar cane and the dissolved sugar simultaneously traverse the diffuser in opposite directions. Two coupled first-order difference equations were derived for the sucrose concentration in the juice and the sucrose concentration in the shredded cane. The difference equations were solved analytically and the results were analysed. Spherical voids can occur in the fibre matrix in the diffuser which is undesirable because it inhibits the extraction process. A model was developed which suggests that voids occur as a result of the decrease with depth in the permeability of the fibre matrix.

\section{Rising water table and seismicity}

The old, now closed, gold mines in the Witwatersrand are being flooded because pumping of water from the mines has ceased. The mines are intersected by geological faults which are stable because of the frictional resistance to movement and the clamping effect of stresses normal to the fault plane. As the level of the water table rises, water will find its way into the geological faults and thus destabilise them. A simple model was presented by Rahab Kgatle which suggests that the time span for water transport into the faults is likely to be relatively short - months rather than years - and that for deep mines the hydrostatic pressure build up within the filled fractures is likely to increase the risk of fault slip. ${ }^{12}$ Slip was predicted to occur along faults not previously prone to slip because of their unfavoured orientation. Under such circumstances, Johannesburg could experience an increase in small seismic events.

\section{An approximate solution to fluid-driven fracture problems}

Hydraulic fracturing - in which water is pumped at high pressure in order to open cracks and fissures in rock - has important applications, for example, in releasing gas and oil from shale deposits deep underground. The equations which describe the fracturing process are highly nonlinear and difficult to solve analytically. Because the fluids used in hydraulic fracturing are generally non-Newtonian, a fracturing fluid with power law rheology was considered. The fractures are long and thin and therefore the injection of fluid into the fracture was modelled by Gideon Fareo using lubrication theory. The model also assumed that the excess fluid pressure in the fracture is proportional to the fracture half-width. Fareo found that the fluid velocity averaged across the width of the fracture varied approximately linearly along the fracture. By assuming that the averaged fluid velocity varies exactly linearly along the fracture, an approximate analytical solution for the evolution of the fracture half-width was derived. The approximate analytical solution agreed well with the numerical solution.

\section{Agent tracking and resource allocation on a network}

Robots now perform many tasks in industry. In the mining and nuclear industries, they are used to enter locations that are too dangerous for humans. Dario Fanucchi presented a talk on a problem in robotics submitted by the CSIR Mobile Intelligent Autonomous Systems.

Significant work has been done within the last 10 years in the context of robotics on the tracking of autonomous agents in complex domains. Fanucchi introduced mathematical models that capture the motion of simple agents that follow certain dynamical rules and move along 
a network. The models were then used to develop a strategy for a separate individual, moving on the same or similar network, to maximise the chance of intercepting one or more agents, possibly weighted by importance. Some applications were considered, notably tracking or intersecting insects or birds with known migration patterns and intercepting moving items on a traffic network.

\section{Looking to the future}

No modeller can resist attempting to extrapolate, so we end by speculating on the future of the trends and issues we have identified.

Ubiquitous modelling is fuelled by accessible modelling tools. Modellers and students of modelling can learn to use a modelling package in a fraction of the time it would take them to study calculus and differential equations. There are, however, trade-offs: mathematics is capable of generalities and insights that are difficult to obtain via computation - hence the quest for mechanistic population models. It is likely that mathematical models will continue to be useful in this regard, providing a 'model of a model': a simplified overview of more detailed computational models (as in the presentation on fluid-driven fractures). However, it is at a more abstract level that mathematics has the most to offer modelling. Computer packages currently allow one to diagram a system model, feed in parameter values, and obtain numerical results for different starting and bounding conditions. What is missing is the ability to understand the meta-properties of the system (stability and resilience, for example). The challenge is to develop modelling packages that routinely provide a meta-analysis.

As powerful modelling tools become easier to use, so they become easier to abuse. There are two responses to this possibility of abuse, both of which are in their infancy. The first is education: there is an urgent need to develop and teach the discipline of modelling. An introductory modelling class should be a requirement for all undergraduate students. The second response is in the development of more intelligent modelling software. Just as word processors routinely check spelling and grammar, so modelling packages could guide and question the user ('why have you chosen such a large time step?') One could imagine software that engages modellers in thinking about their model world, suggesting alternative modelling approaches, designing assumption analyses, and providing explanations to the user of the model. Models that are used on a regular basis could monitor their performance, update their parameter values, and suggest changes to the structure of the model. Modelling education and more intelligent software will be symbiotic.

Finally, the diversity of papers presented at this symposium gives a glimpse of the future of modelling in South Africa. Traditional areas of strength such as geomechanics, ecology and resource management will continue to flourish and will likely export ideas and modelling expertise - models that involve stakeholders in resource management, for example, provide a paradigm for all sorts of policy decisions. Modelling successes in the Mathematics in Industry initiative will in turn generate new interests and opportunities. South African involvement in the Square Kilometre Array radio telescope has already led to an upsurge in interest in astronomy and astrophysics; observations from the telescope will create a fertile environment for model development and model testing. Modelling skills developed in one area of application prepare a modeller to work in other areas of application. It follows that providing modelling opportunities and apprenticeships in all of the above will train modellers to deal with the problems of the future, such as those engendered by sustainable energy production and climate change.

\section{Acknowledgements}

D.P. Mason and M. Sears thank the Research Committee of the Faculty of Science of the University of the Witwatersrand, Johannesburg, for a grant to run the symposium. We also thank the symposium presenters for their input.

\section{References}

1. Mason DP, Wilson DB. Papers to honour A.M. Starfield on his sixtieth anniversary. S Afr J Sci. 2002;98:441-502.

2. Starfield AM, Salter RM. Thoughts on a general undergraduate modelling course and software to support it. Trans R Soc S Afr. 2010;65:116-121. http://dx.doi.org/10.1080/0035919X.2010.513172

3. Demarcq H, Richardson AJ, Field JG. Generalised model of primary production in the southern Benguela upwelling system. Mar Ecol Prog Ser. 2008;354:59-74. http://dx.doi.org/10.3354/meps07136

4. Williamson R, Field JG, Shillington FA, Jarre A, Potgieter A. A Bayesian approach for estimating vertical chlorophyll profiles from satellite remote sensing: Proof-of-concept. ICES J Mar Sci. 2011;68:792-799. http://dx.doi. org/10.1093/icesjms/fsq169

5. Smith MD, Jarre A. Modelling regime shifts in the southern Benguela: $A$ frame-based approach. Afr J Mar Sci. 2011;33(1):17-35. http://dx.doi.org /10.2989/1814232X.2011.572334

6. Starfield AM, Jarre A. Interdisciplinary modelling for an ecosystem approach to management in marine social-ecological systems. In: Ommer RE, Perry RI, Cochrane K, Cury P, editors. World fisheries: A social-ecological analysis. Oxford: Wiley-Blackwell; 2011. p. 105-119. http://dx.doi. org/10.1002/9781444392241.ch6

7. Owen-Smith N. Accommodating environmental variation in population models: Metaphysiological biomass loss accounting. J Anim Ecol. 2011;80:731-741. http://dx.doi.org/10.1111/j.1365-2656.2011.01820.x

8. Richardson FD, Hoffman MT, Gillson L. Modelling the complex dynamics of vegetation, livestock and rainfall in a semi-arid rangeland in South Africa. Afr J Range For Sci. 2010;27:125-142. http://dx.doi.org/10.2989/10220119.2 010.520676

9. Mathematics in Industry Study Group 2013 [homepage on the Internet]. c2013 [cited 2014 May 13]. Available from: http://www.wits.ac.za/ conferences/misgsa2013.

10. Herbst RS, Qadir A, Momoniat E. The effects of mass on the radiation of a relativistically rotating neutron star. New Astronomy. 2013;25:38-44. http:// dx.doi.org/10.1016/j.newast.2013.03.010

11. Breward C, Hocking G, Ockendon H, Please C, Schwendeman D. Modelling the extraction of sugar from sugar cane in a diffuser. Proceedings of the Mathematics in Industry Study Group 2012. Johannesburg: University of the Witwatersrand; 2012. p. 31-57.

12. Fowkes ND, Hocking G, Please CP, Mason DP, Kgatle MR. Rising water table and seismicity. Proceedings of the Mathematics in Industry Study Group 2012. Johannesburg: University of the Witwatersrand; 2012 p. 1-19. 